\title{
Scanning-Based Human-Computer Interaction Using Intentional Muscle Contractions
}

\author{
Torsten Felzer, Rainer Nordmann, and Stephan Rinderknecht \\ Department of Mechatronics in Mechanical Engineering \\ Darmstadt University of Technology \\ Petersenstr. 30, D-64287 Darmstadt, Germany \\ \{felzer,nordmann, rinderknecht\}@mim.tu-darmstadt.de
}

\begin{abstract}
It has already been shown in the past that it is possible to leverage tiny muscular contractions produced at will (e.g., by frowning) in order to give someone complete control over a PC 11. The underlying interaction technique is ideal for persons with severe motor impairments who are in need for an alternative, non-standard way to operate a computer. This paper deals with a scanning-based computer application of that approach to enable its user to control the immediate environment, e.g., by making a phone call, toggling the lights, or sending particular Infra-Red (IR) remote signals. Although the software is primarily targeted at people with disabilities, it is ready - and (in certain situations) even expected - to be used by able-bodied individuals as well. A user study evaluating the remote control module of the system has been conducted with twelve non-impaired subjects, and the results are discussed herein.
\end{abstract}

keywords: Human-computer interaction, bio-signal interfaces, scanning, hands-free access, universal remote control, Speech API (SAPI).

\section{Introduction}

Technological progress is visible everywhere nowadays, in particular concerning the development of personal computers. For example, a modern graphics card manages a multiple of the memory capacity of a high-end hard disk drive from the late 1980 s - at only a fraction of the cost. However, it is very interesting that the standard way to interact with a computer is basically the same as it was half a century ago. Of course, the typewriter-style keyboard was supplemented with a pointing device (a necessary step with the advent of GUI-based - as opposed to pure text-based - operating systems), but the operation still requires - in the standard case - the usage of the hands. This is also true for many computermediated (or "computer-like") devices, such as a telephone or an infra-red remote control.

Unfortunately, not everyone is able to reliably employ the hands. To be able to control a computer (and consequently the immediate environment), many persons with physical disabilities rely on appropriate alternative interfaces. Moreover, an able-bodied person may not want to leave the exercising machine when

C. Stephanidis (Ed.): Universal Access in HCI, Part II, HCII 2009, LNCS 5615, pp. 509-518, 2009.

(C) Springer-Verlag Berlin Heidelberg 2009 
listening to music while working out (just to pick up the remote to skip to the next song), or probably he/she feels uncomfortable with touching the cordless phone with wet hands (when a call is coming in while lying in the bathtub).

The next section discusses common ("hands-free") alternatives in use so far. This is followed by a closer look at a certain muscle-based input principle, which is applied to an environment control system in section 4, while section 5 presents a user study with twelve able-bodied participants, evaluating an integral part of that system. A brief conclusion is given in section 6

\section{Related Work}

As for hands-free computer access, persons who do not have any articulation problems can benefit from solutions based on speech recognition, i.e., "talk to their computer" (e.g., [2]). Eye trackers comprise a second group of (mostly rather expensive) alternatives (e.g., 3). Switch-activated devices often work in conjunction with a particular scanning software (e.g., [4]), where the computer suggests output actions by cyclically highlighting a number of available options, and the user may select the highlighted option by activating a certain switch (also [5]).

A fourth, very large group of alternatives is given by so-called bio-signal interfaces: those systems monitor the time series of a certain bodily function of the user and generate corresponding output commands in response to specific patterns in the monitored signal - willfully issued by the user [6]. An important representative is an EEG-based Brain-Computer Interface (BCI) which analyzes the on-going brainwaves of a subject (e.g., 7]). What makes this very popular as a concept helping persons with physical disabilities is that it requires mental activities only. The major disadvantage of BCI's is that EEG recording is very slow and sensitive (e.g., 8 $)$.

The input idea detailed in this paper represents a mixture between a bio-signal interface and a single-switch device. On the one hand, the muscular activity of a single dedicated muscle is inspected, in order to detect intentional contractions of that muscle. On the other hand, the contraction events are used in analogy to the activation of a switch, i.e., processed in a scanning-like fashion. The same input concept has already been implemented in a mouse emulator (see fig. 1) allowing its user to control a PC (or a computer-mediated device) just by frowning (without the need to use the hands) [1]. However, as that tool is optimized for mouse actions, only its data acquisition engine can be reused here.

\section{Input Principle}

Instead of any manual interaction, the technique focused here relies on tiny contractions of an arbitrary muscle of choice. To detect those input signals, the amplitude of the muscular activity of the chosen muscle - recorded and amplified with the help of a piezo-based sensor - is compared to a user-dependent threshold. Exceeding the threshold triggers a contraction event. 

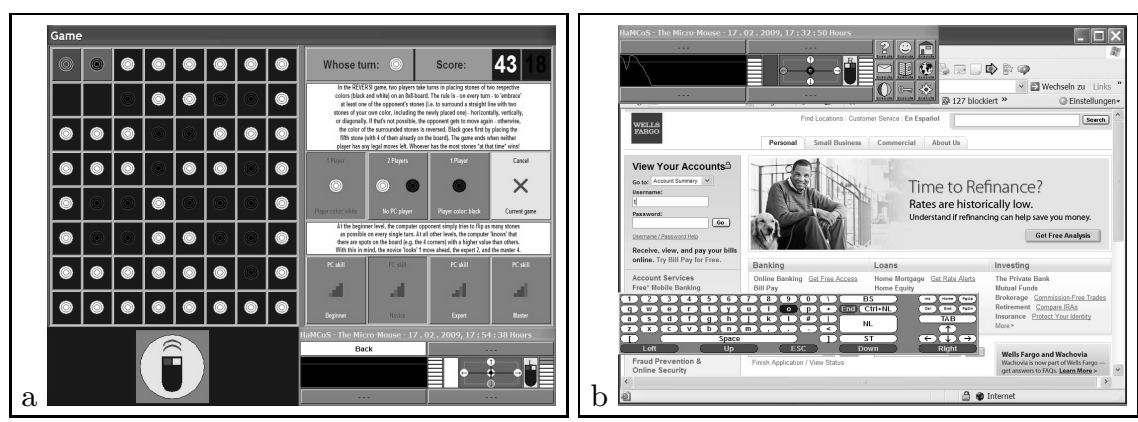

Fig. 1. The "HAnds-free Mouse COntrol System" (HaMCoS) allows its user to operate a PC completely, only by issuing tiny muscle contractions. a) Various framework applications each deal with a specific task - the application shown here is for playing a certain board game against the computer (it is intended to provide a hands-on approach for getting familiar with the system); b) with HaMCoS, it is also possible to employ standard tools in a non-standard way, e.g., logging in to a banking site (displayed in an ordinary web browser) can be done by opening a special on-screen keyboard.

Depending on the amount of time between two contraction events, the HaMCoS tool mentioned above discerns a Single (SC) and a Double Contraction (DC). Those two input events are processed by switching among the states of a Finite State Machine (FSM), and the currently activated state governs mouse pointer movement. As a result, HaMCoS can emulate a mouse device, and its user just needs to issue SC's and DC's.

In contrast to automatic scanning (where the highlight automatically cycles to the next option after a certain time period has elapsed), HaMCoS basically implements some form of self-paced scanning, in that it cycles to the next pointer movement direction (in a given order) upon detection of explicit contraction events. The application detailed in the next section reverts to conventional (automatic) scanning with contraction signals to select the highlighted option.

\section{Environment Control Application}

An Environment Control System (ECS) has been implemented (in $\mathrm{C}++$, under Windows $(\mathrm{XP})$ as a target application, capable of processing the muscle-related input signals. The ECS (depicted in fig. 2) basically offers four modules, e.g., turning the PC into a comfortable telephone set or into a Universal Remote Control (URC). Each module is associated with up to 32 buttons (arranged in an $8 \times 4$ grid) which are activated using a special variant of row-column scanning - or optionally using a manual mouse to cut short the entry of longer button sequences for able-bodied users.

Only one of the four module displays is active at any given time. When the user triggers the selection of a button of that module, the display cycles through the representations symbolized in fig. 3. At the top level (following the selection of another button), the four rows are scanned from top to bottom, i.e., the 


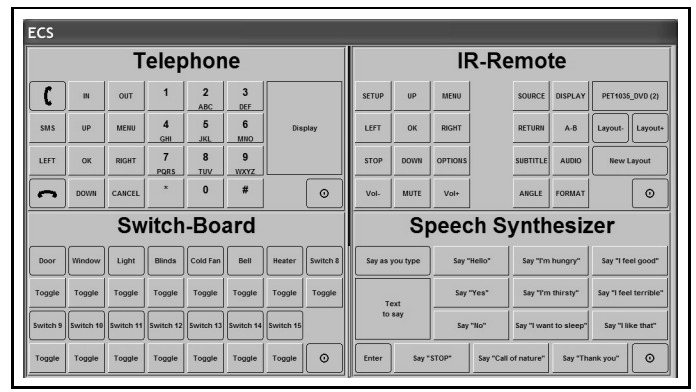

Fig. 2. The Environment Control System comprises four individual modules

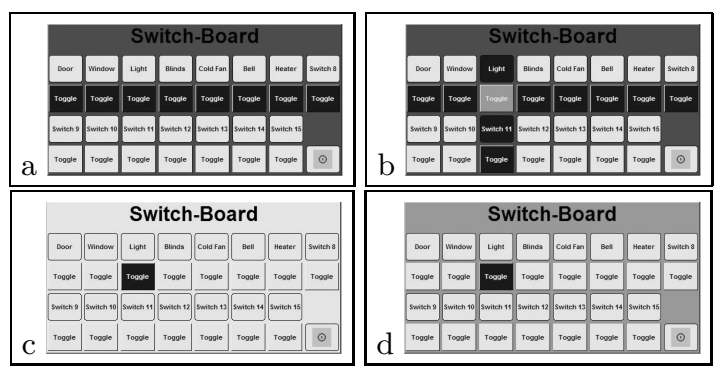

Fig. 3. Different phases during the selection cycle of any button are accompanied by specific display layouts. This is illustrated using the example of toggling the lights: a) row scan; b) column scan; c) confirmation period; d) notification period.

(eight) buttons in a specific row are highlighted, and the highlight advances to the next row (cyclically) after the scan-delay 1 . Following a contraction while the desired row is highlighted, the eight columns are scanned left to right (and the "intersecting" button is particularly marked). A second contraction pre-selects a button, but to make the system fault-tolerant, the associated action will not be generated unless the user confirms the selection with a third contraction in the subsequent period of length $\tau$. A successful confirmation is answered with a notification (as depicted in fig. 3 d)). The entire process starts anew with a row scan in either case.

\subsection{Functionality}

The main purpose of the ECS is to provide an accessible interface for persons with severe motor impairments. In this respect, each of the four modules deals with a different aspect of everyday life.

The first (top left) module is for making telephone calls, answering incoming calls, managing phone numbers as well as call histories, or composing text

${ }^{1}$ For the time being, the scan-delay is set to $\tau=1.0 \mathrm{~s}-$ it shall be made adjustable in future versions. 


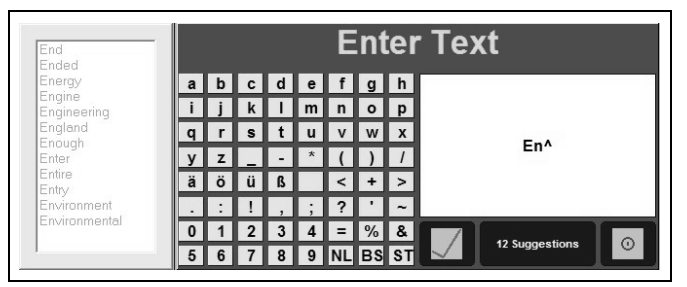

Fig. 4. The editor routine - shared by all four modules - helps the user in saving "keystrokes" by implementing Word Prediction (WP). Here, entering "En" leads to the 12 suggestions displayed in the box on the left.

messages - its purpose is to simply replace the usual telephone with a hands-free alternative 2 .

Whenever the user wishes to enter a text message ("SMS"), the module display is temporarily replaced by the editor routine depicted in fig. 4. In addition to an on-screen keyboard with $8 \times 8$ "character buttons" (to be selected just as any other button) on the left, the editor presents the entered text as well as some miscellaneous buttons on the right (e.g., for confirming or discarding the input). In order to help the user save keystrokes, the editor suggests prediction candidates of the currently "typed" word based on lists of frequently used terms 9]. Closing the editor with the "OK" button results in on-line adaptation of the personal frequency list. The same editor routine is invoked for all text entry requirements in the other modules as well.

After the installation of switchable power outlets, the switch-board module on the lower left can be used to turn suitable appliances on or off. Possible target devices include lamps, a fan, a heater, blinds, or an electric door opener.

The top-right module allows the user to transmit pre-recorded IR signals without the need to employ the hands. It turns the PC into a URC, and, once programmed (preferably to be done by an able-bodied caregiver) it can be of great help for a motor-impaired person. As this module is in the focus of the user study presented in the next section, it is described in more detail below.

The fourth module enables a speech impaired user to "speak with the computer's voice". Applying the Windows@ SAPI, this component not only offers to pronounce standard, pre-determined utterances (like "Yes", "No", or "Thank You"), but also arbitrary user-entered sentences. The word prediction feature makes it even possible to have simple conversations via PC.

\subsection{Universal Remote Control}

The display of the "IR-Remote" module is dominated by a layout representing (a part of) an original remote control with (up to) 24 buttons associated with

${ }^{2}$ It should be noted that only the user interface of this module is finished at this point. There are precise ideas for realizing the functionality, but the actual implementation remains a task for the near future. 

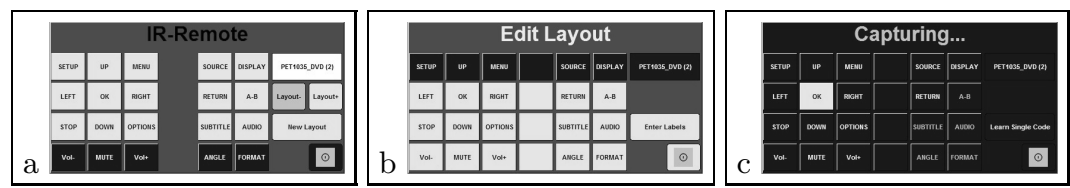

Fig. 5. The remote control module runs in two modes: the default transmit mode (a) and a configuration mode offering to edit the button labels (b) or to capture IR data (c).

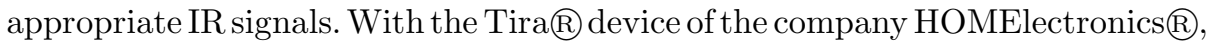
the software is able to replace the corresponding remote.

The user (or its able-bodied assistant, in case of a disabled user) can "copy" the remote of any appliance by creating a new layout, labeling the 24 IR code buttons, and assigning suitable IR signals to them (see fig. 5). There is no limit as to the number of layouts to be created - new layouts are stored on hard disk and (so far) accessed linearly.

\section{Evaluation Study}

A user study evaluating the remote control module of the environment control system described in the previous section has been conducted with two goals in mind. First, it was intended to verify the general usefulness of the software. The second objective was to investigate how able-bodied users react to this software.

\subsection{Participant Recruitment and General Organization}

It was decided to look for possible study participants among the non-impaired members of the technical staff at the authors' university. It is true that that group is surely not representative of all non-impaired people, but as they are using a computer at work, they are able to estimate the value of a software from a rather comprehensive perspective.

Twelve subjects (one female) accepted our invitation to take part in the study. The ages ranged between 26 and 38 years with an average of 28.4 years (standard deviation 3.4). The average usage of IR-controlled devices was 2.9 hours per week (standard deviation 4.8), while five participants did not use any IR-controlled device at all on a regular basis. All twelve were already familiar with the general concept of a URC, though none of them utilized one him-/herself.

The user contribution was divided into three parts: one practical session (per subject!) and two questionnaires, one before and one after the practical session. The pre-evaluation questionnaire asked for certain demographic data as well as the participants' prior exposure to IR-controlled devices. It also requested informed consent.

The post-evaluation questionnaire asked about the (subjective) opinions of the participants regarding the software - after they had a chance to emulate a particular remote with the tool. Response options corresponded to seven-point Likert-style scales. 


\subsection{Practical Sessions}

The practical sessions involved the experimental setup depicted in fig. 6. Unfortunately, the PHILIPS $\cap$ device has two major drawbacks (with quality being its main advantage). On the one hand, it comes with an alternating remote control. This means that two consecutive keypresses on the same button of the remote control do not result in the transmission of the same IR code. Instead, the remote stores two codes for each button, say, one of some type A as well as one of type B, and the remote constantly alternates between A and B for each keypress. This is no problem as long as one is using the original remote, since the receiving unit also alternates in the code type to expect. However, it makes life extremely unpleasant for users of universal remotes, since they not only have to assign two URC buttons to every remote control function, they also have to do the alternation "by hand".

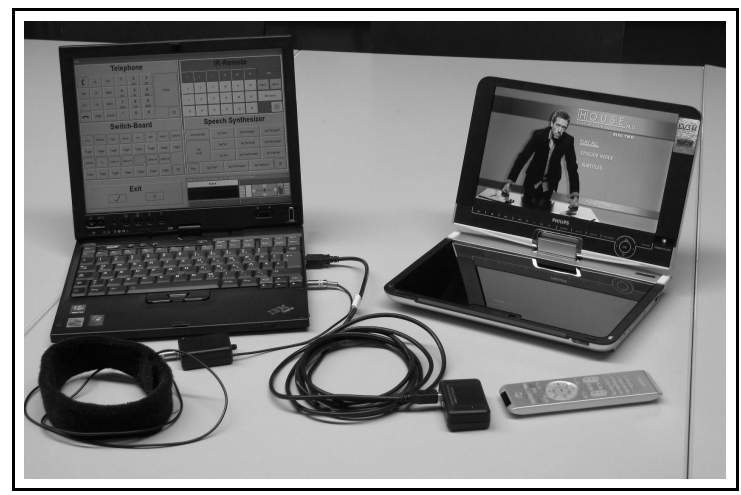

Fig. 6. The experimental setup involved an $I B M \cap X 41$ tablet $P C$ with a headband sensor connected to the microphone input (left) and a PHILIPS® PET 1035 portable DVD player as well as a USB-driven Tira® device (right).

The second drawback relates to the timeout of certain menus (e.g., for configuring the subtitle settings). An open menu will automatically disappear, if the unit does not receive a code of the expected type for more than about 14 seconds. This is particularly frustrating if one has managed to descend three levels, but then just needs half a second too long for triggering the finalizing "Play" (as the menu has to be reopened at the top level) 3 .

The individual practical sessions began with a detailed explanation of the setup and the used software tool. The participant's task was then to emulate (partially) the remote of the DVD player - this involved the following four phases:

${ }^{3}$ The DVD player originally designated for the study broke the day before the scheduled practical sessions - a replacement had to be obtained rapidly ... 
1. assigning the 12 most important remote control functions to the IR code buttons of an own layout, i.e., labeling the 24 buttons, 4 ,

2. learning the IR code of each button, i.e., capturing the signals issued by pressing corresponding keys on the original remote,

3. verifying that the signals were captured correctly, and

4. applying the hands-free input method to the newly created layout.

Researchers recorded the duration of each phase, and they were also ready to answer questions or to give hints when requested. However, the subject was encouraged to perform all subtasks without further guidance (and it was their decision when to end a phase and move on to the next).

\subsection{Results and Discussion}

Since every participant was asked to copy the same 12 remote control functions, the resulting layouts looked quite similar - fig. 7 shows a typical representative. The numerical results regarding the practical sessions and the post-evaluation questionnaires are presented in tables 1 and 2.

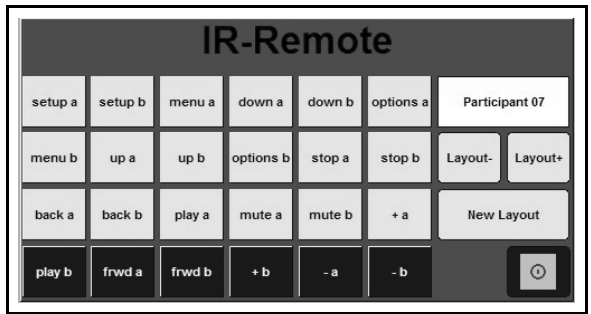

Fig. 7. The resulting layouts resemble each other - that of Participant 07 is typical

It can be seen in table 1 that capturing the 24 IR codes (D2) can be done relatively fast with the program as opposed to labeling the buttons. The (mostly) large $D 4$ value suggests that participants were quite satisfied being able to operate the DVD player hands-free. This satisfaction can also be deducted from table 2 (with positive scores for almost every question, particularly the one about the social value). The scores might be even better, if the chosen equipment did not have the drawbacks mentioned above6.

The only exception is question $Q_{4}$ : participants doubt they will use the program again. There might be three reasons for this. First, having a motor-impaired family member would greatly increase the usage probability, but this was no requirement when recruiting study participants. Second, an able-bodied person

\footnotetext{
${ }^{4}$ The mouse was used here on the on-screen keyboard of the editor (without WP).

${ }^{5}$ Many participants wished to use the keyboard rather than mouse-based shortcuts for labeling - in the meantime, this functionality was added.

${ }^{6}$ An optional support of alternating remotes was implemented directly after the study.
} 
Table 1. Objective, measurable results. The first (value) line in this table denotes the number of mouse clicks each participant performed while labeling all of the buttons of "her/his" layout (which relates to the total number of characters), D1 is the amount of time needed for that, and Rate is the quotient of those two values. D2, D3, and D4 stand for the time each participant spent capturing the IR codes of all buttons, verifying (testing) the stored codes (with the mouse), and evaluating the final layout with the help of the alternative (hands-free) input method, respectively. All duration values are given in minutes (rounded to the nearest integer).

\begin{tabular}{|c|c|c|c|c|c|c|c|c|c|c|c|c|c|c|}
\hline \multirow[t]{2}{*}{ Feature } & \multicolumn{12}{|c|}{ Participant } & \multirow[t]{2}{*}{ Avg. } & \multirow{2}{*}{$\begin{array}{l}\text { Std. } \\
\text { Dev. }\end{array}$} \\
\hline & 01 & 02 & 03 & 04 & 05 & 06 & 07 & 08 & 09 & 10 & 11 & 12 & & \\
\hline "Keystrokes" & 263 & 201 & 248 & 221 & 180 & $\overline{229}$ & 192 & 248 & 165 & 219 & $\overline{126}$ & 196 & 207.3 & $\overline{39.0}$ \\
\hline D1 (Labels) & 15 & 12 & 15 & 15 & 21 & 25 & 10 & 10 & 8 & 11 & 10 & 12 & 13.6 & 4.9 \\
\hline Rate [kspm] & 17.5 & 16.7 & 16.5 & 14.7 & 8.5 & 9.1 & 19.2 & 24.8 & 20.6 & 19.9 & 12.6 & 16.3 & 16.3 & 4.6 \\
\hline D2 (Capture) & 3 & 2 & 3 & 3 & 3 & 3 & 2 & 2 & 3 & 3 & 6 & 3 & 3.0 & 1.0 \\
\hline$\overline{\text { D3 (Test) }}$ & 5 & 2 & 3 & 4 & 12 & 9 & 2 & 3 & 7 & 7 & 2 & 2 & $\overline{4.8}$ & 3.2 \\
\hline D4 (Altern.) & 12 & 11 & 12 & 14 & 7 & 5 & 19 & 6 & 16 & 7 & 27 & 16 & 12.6 & 6.3 \\
\hline
\end{tabular}

Table 2. Subjective evaluation (scores on Likert-type seven-point scales). The five Likert-type questions were as follows: Q1. How easy was it to use the program (1=very easy; $7=$ very hard); Q2. How enjoyable was it to use the program (1=very enjoyable; $7=$ very annoying); Q3. How would you rate your sympathy towards the program (1=liked it; $7=$ hated it); Q4. How probable is it that you want to use the program again (1=very probable; $7=$ very improbable); Q5. How would you rate the (social) value of the program $(1=$ very valuable; $7=$ no value at all $)$.

\begin{tabular}{|c||c|c|c|c|c|c|c|c|c|c|c|c|c|c||c|c|}
\hline Feature & \multicolumn{10}{|c|}{ Participant } & Avg. & $\begin{array}{c}\text { Std. } \\
\text { Dev. }\end{array}$ \\
\hline \hline Q1 (Easy) & 3 & 3 & 2 & 4 & 4 & 3 & 3 & 3 & 2 & 2 & 3 & 3 & 2.9 & 0.6 \\
\hline Q2 (Enjoyable) & 2 & 5 & 3 & 3 & 3 & 2 & 2 & 2 & 2 & 3 & 4 & 2 & 2.7 & 0.9 \\
\hline Q3 (Sympathy) & 2 & 2 & 3 & 3 & 2 & 1 & 2 & 3 & 1 & 3 & 3 & 2 & 2.2 & 0.7 \\
\hline Q4 (Again?) & 6 & 4 & 4 & 2 & 2 & 6 & 6 & 5 & 3 & 5 & 6 & 6 & 4.5 & 1.5 \\
\hline Q5 (Value) & 2 & 2 & 3 & 3 & 1 & 1 & 3 & 2 & 1 & 2 & 1 & 1 & 1.8 & 0.8 \\
\hline
\end{tabular}

probably prefers (and expects) features our program does not have (other than, e.g., providing hands-free access). And finally, as the usage of IR-controlled devices is rather modest among the members of the study group, it is not too probable that they will use any URC software in the first place.

\section{Conclusion}

An environment control system intended to enable someone with severe physical impairments to control her/his immediate surroundings, e.g., by turning on the lights or switching channels on the TV has been described. The tool merely relies on intentional muscle contractions as input signals - for instance, the system asks for no more physical contribution than simply frowning. The software proved to 
be satisfactory in a user study with able-bodied subjects, yet it can hardly be considered a general alternative for non-impaired individuals (i.e., not assisting someone with a disability). Another user study investigating the reactions of persons with motor impairments is a necessary next step.

The same input idea has already been applied to the PC operation tool HaM$\mathrm{CoS}$ as well as a system controlling an electric powered wheelchair [10]. All three systems are constantly being extended and ultimately combined with HaMCoS being the common platform, which greatly adds to the independence of a severely disabled person.

Acknowledgments. This work is supported by DFG grant FE 936/3-1"The AID package - An Alternative Input Device based on intentional muscle contractions".

\section{References}

1. Felzer, T., Fischer, R., Grönsfelder, T., Nordmann, R.: Alternative control system for operating a PC using intentional muscle contractions only. In: Online-Proc. CSUN Conf. (2005)

2. Assistive Technology \& Accessibility at Oklahoma State University - Dragon NaturallySpeaking 9.0, http://access.it.okstate.edu/content/view/22/

3. Wobbrock, J.O., Rubinstein, J., Sawyer, M., Duchowski, A.T.: Not Typing but Writing: Eye-based Text Entry Using Letter-like Gestures. In: Proc. COGAIN 2007, pp. 61-64 (2007)

4. Baljko, M., Tam, A.: Motor input assistance: Indirect text entry using one or two keys. In: Proc. ASSETS 2006, pp. 18-25. ACM Press, New York (2006)

5. Steriadis, C.E., Constantinou, P.: Designing human-computer interfaces for quadriplegic people. ACM Trans. Comput. Hum. Interact. 10(2), 87-118 (2003)

6. Felzer, T.: Verwendung verschiedener Biosignale zur Bedienung computergesteuerter Systeme (Using various kinds of bio-signals for controlling computermediated systems). Ph.D. Thesis (German). Wissenschaftl. Verlag Berlin (2002)

7. Birbaumer, N., Hinterberger, T., Kübler, A., Neumann, N.: The thoughttranslation device (TTD): Neurobehavioral mechanisms and clinical outcome. IEEE Trans. Neural Syst. Rehabil. Eng. 11(2), 120-123 (2003)

8. McFarland, D.J., Sarnacki, W.A., Vaughan, T.M., Wolpaw, J.R.: Brain-computer interface (BCI) operation: Signal and noise during early training sessions. Clinical Neurophysiology 116, 56-62 (2005)

9. Felzer, T., Nordmann, R.: Speeding up hands-free text entry. In: Proc. CWUAAT 2006, pp. 27-36. Cambridge University Press, Cambridge (2006)

10. Felzer, T., Nordmann, R.: Alternative wheelchair control. In: Proc. RAT 2007, pp. 67-74. IEEE Computer Society Press, Los Alamitos (2007) 